\title{
La reorientación del ciclo de vida del área turística. \\ El caso de Bahías de Huatulco, Oaxaca (México).
}

\author{
Víctor Manuel López Guevara \\ Máster en Dirección y Planificación del Turismo \\ Universidad de Alicante \\ guevara@huatulco.umar.mx
}

\section{RESUMEN}

La publicación del modelo de Richard Butler (1980) referente al Ciclo de Vida del Área Turística (CVAT) ha generado un debate permanente en torno a la evolución de los destinos vacacionales. Con base en la revisión de diversos estudios que contrastan y cuestionan la validez del CVAT, en este trabajo se formula un esquema alternativo de análisis mediante el cual se realiza el diagnóstico de las fases de desarrollo y situación actual de un centro planificado de turismo litoral ubicado en la costa sur de México: Bahías de Huatulco.

A través del caso de estudio se evidencia la pertinencia del modelo propuesto, se determina que el centro turístico en cuestión -aún cuando no ha alcanzado su máximo grado de desarrollo-, se encuentra en medio de una fase de reorientación y se anota una serie de propuestas para revitalizar la actividad turística.

Palabras clave: Turismo litoral, ciclo de vida, estrategias de reestructuración, Bahías de Huatulco. 


\title{
The reorientation of the life cycle of the tourist area
}

The case of Huatulco, Oaxaca (Mexico).

\author{
Víctor Manuel López Guevara \\ Master's Degree in Tourism Management and Planning \\ University of Alicante \\ guevara@huatulco.umar.mx
}

\section{ABSTRACT}

The publication of Richard Butler's Touristic Area Life Cycle model (TALC) in 1980 led to an intensive and ongoing discussion regarding development of tourist destinations. On the basis of some major subsequent studies which question the validity of the TALC, this thesis will look into an alternative model. This is done by identifying and analyzing the current situation as well as different stages of development of a planned tourism destination located in the coastal region of southern Mexico: Bays of Huatulco.

The case study demonstrates the appropriateness of the proposed model. Furthermore, it is shown that the analyzed region Bays of Huatulco has not yet reached its maximum degree of development and is currently on the so-called 'reorientation' stage. Some further recommendations to revitalize the tourism activity in this tourism resort are also provided.

Key words: Coastal Tourism, Life Cycle, Restructuring Strategies, Bays of Huatulco. 


\section{INTRODUCCIÓN}

El mapa mundial del turismo litoral es extenso y en él se distinguen áreas de diferente origen generacional que buscan posicionarse en el mercado (Artigues, 2001:108). En medio de este escenario, los destinos turísticos en situación comprometida suelen aplicar diversas medidas para reorientar su evolución y recuperar su competitividad. Las actuaciones implementadas pueden ser llamadas de diferentes formas (reestructuración, renovación o reconversión), no obstante, en todas los casos suelen incidir sobre el mejoramiento de la oferta, la búsqueda de la eficiencia empresarial y la intervención sobre la estructura territorial (Getz, 1992; Vera et al., 1997; Camisón y Monfort, 1998; Donaire y Mundet, 2001; Johnston, 2001; Agarwal, 2002; Antón, 2004 y Vera y Rodríguez, 2010).

A pesar del nivel de comprensión alcanzado en torno a la evolución de los destinos y a la relación que guarda ésta con la aplicación de acciones de reorientación, el interés por su estudio se mantiene vigente por dos razones: 1) por la constante competencia que obliga a la permanente reformulación de estrategias y 2) porque el turismo litoral ocupa un lugar central en la economía de varios países, entre ellos México.

A nivel mundial los estudios suelen enfocar su atención en los destinos con mayor grado de consolidación. Esta tendencia también se presenta en México y de ella dan testimonio algunos trabajos referidos a los casos de Acapulco (Barbosa, 2007), Cancún (Moncada, 2008), Mazatlán (Santamaría y Barbosa, 2008) y Puerto Vallarta (Virgen, 2009). Otros destinos mexicanos, como los planificados por el gobierno federal desde los años ochenta, no han sido objeto del mismo interés, presumiblemente porque aún no manifiestan señales de consolidación o de saturación.

Sin embargo, los destinos no consolidados presentan retos cuya gestión puede resultar igual de apremiante que la solución a las problemáticas en las áreas saturadas. El caso de Bahías de Huatulco es ejemplo de ello. De acuerdo con el Plan Maestro de este destino, al finalizar la primera fase de su desarrollo (1984-1988) tendría que haber reportado 1.300 cuartos en operación, sin embargo, al cumplirse el plazo la oferta de alojamiento totalizaba 592 habitaciones. Durante los siguientes años el crecimiento de la oferta mantuvo un ritmo menor al proyectado y así, en 2000 , fecha en la que culminó la segunda etapa de desarrollo, el número total de cuartos disponibles era de 2.160 equivalentes al $34,1 \%$ de la meta comprometida. Como consecuencia de la situación antes mencionada el destino en cuestión experimentó problemas para atraer líneas aéreas, touroperadores, empresas de ocio y, en consecuencia, turistas.

El caso de Bahías de Huatulco pone de manifiesto la pertinencia de examinar la evolución seguida por los centros turísticos no consolidados. En este sentido la presente investigación se propone tres objetivos: caracterizar el ciclo de vida del destino, identificar si en él se manifiesta estrategias de reorientación y, en caso de que así sea, describirlas y proponer medidas que junto a las detectadas permitan coadyuvar a la dinamización del ciclo de vida del área turística estudiada. 
Este trabajo se estructura en tres secciones. En la primera se enuncian algunas posiciones teóricas de partida y se define un modelo de referencia para conducir el análisis. En la siguiente sección se presentan los resultados obtenidos y en la tercera se indican las conclusiones derivadas del estudio.

\section{MODELOS PARA ABORDAR EL CICLO DE VIDA}

\subsection{Tres puntos de partida: Miossec, Butler y Chadefaud}

De acuerdo con Vera et al. (1997:233), desde finales de los años setenta se ha puesto en evidencia el papel del turismo como instrumento promotor del desarrollo territorial. A partir de entonces se han ensayado diversos modelos que buscan explicar dos aspectos: la evolución que sigue el desarrollo de los destinos y la forma en la cual las áreas turísticas, vistas como productos, pueden mantenerse vigentes a lo largo del tiempo. Entre los modelos más representativos destacan las propuestas elaboradas por Miossec (1977), Butler (1980) y Chadefaud (1987).

Con base en la discusión realizada por López (2010:29-31) acerca de los tres modelos, se decidió seleccionar la propuesta del Ciclo de Vida del Área Turística (CVTA) de Richard Butler para guiar el análisis de Bahías de Huatulco. Tres razones motivaron la elección:

Primero. El modelo de Miossec está pensado para analizar la evolución del turismo a escala región, situación que escapa de los objetivos de la presente investigación.

Segundo. Si bien es claro que la propuesta de Chadefaud se encuentra dotada de un interesante andamiaje teórico-conceptual que permite entender la evolución de las periferias turísticas, no se ha logrado identificar en ella, a través de las fuentes consultadas, un listado de variables que permitan evaluar el grado de correspondencia entre el mito y el espacio-producto. Sin ese referente se vuelve difícil determinar la etapa evolutiva de los destinos.

Tercero. EI CVAT ha sido debatido intensamente a nivel internacional tanto en lo concerniente a sus bases teóricas, limitaciones conceptuales y restricciones operativas. López (2010:38-39) sintetiza las críticas hechas al modelo entre las cuales figuran la medición del desarrollo turístico con base en el incremento histórico de turistas (lo cual parece sugerir que la masificación es la meta perseguida), las dificultades que supone obtener información fiable y estandarizada, la falta de criterios claros para marcar límites entre una etapa y otra del ciclo, problemas vinculados a la escala de análisis y la concepción de modelo evolutivo como una secuencia que siempre sigue un orden definido. A partir del reconocimiento de las limitaciones antes señaladas se facilita la elaboración de modelos derivados o alternativos que permitan el desarrollo de aproximaciones analíticas adecuadas a la casuística de cada destino turístico. 


\subsection{Un modelo de CVAT para Bahías de Huatulco}

Una vez revisadas las diferentes observaciones hechas al modelo de Butler por autores como Getz (1992), Cooper (1994), Cohen y Shoval (2004), Vera (2004) y Diedrich y García (2009); así como las modificaciones que al mismo han propuesto Priestley y Mundet (1998), Johnston (2001) y Agarwal (2002 y 2006), se elaboró una nueva versión para estudiar la evolución de Bahías de Huatulco (figura 1). En ella se incorporaron tres modificaciones, a saber:

1a) La sustitución del número de turistas como variable de referencia. En su lugar se propone el manejo de la ocupación hotelera debido a que esta variable sugiere el nivel de competitividad alcanzada por el destino a lo largo de todo el año,

2a) La incorporación de la noción de Chadefaud (1987, citado en Callizo, 1989:40-43) sobre el mito y el espacio-producto como elemento auxiliar para distinguir fases evolutivas y

3a) Debido a que Bahías de Huatulco es un destino ex novo, fueron consideradas las anotaciones de Haywood (1986, citado en Vera et al. 1997:239) relacionadas a los complejos turísticos de origen instantáneo y, en consecuencia, se eliminaron las dos primeras fases manejadas por Butler (exploración e involucramiento). La fase de estancamiento fue sustituida por la de saturación para guardar correspondencia con las etapas de desarrollo contempladas en la planificación oficial del destino. Los indicadores considerados para identificar cada una de las etapas guardan correspondencia con los planteamientos de Butler (1980), Cooper (1994), Johnston (2001) y Agarwal (2002 y 2006). Para una descripción detallada consúltese López (2010: 49-51).

Figura 1. Modelo alternativo para estudiar el CVAT de Bahías de Huatulco

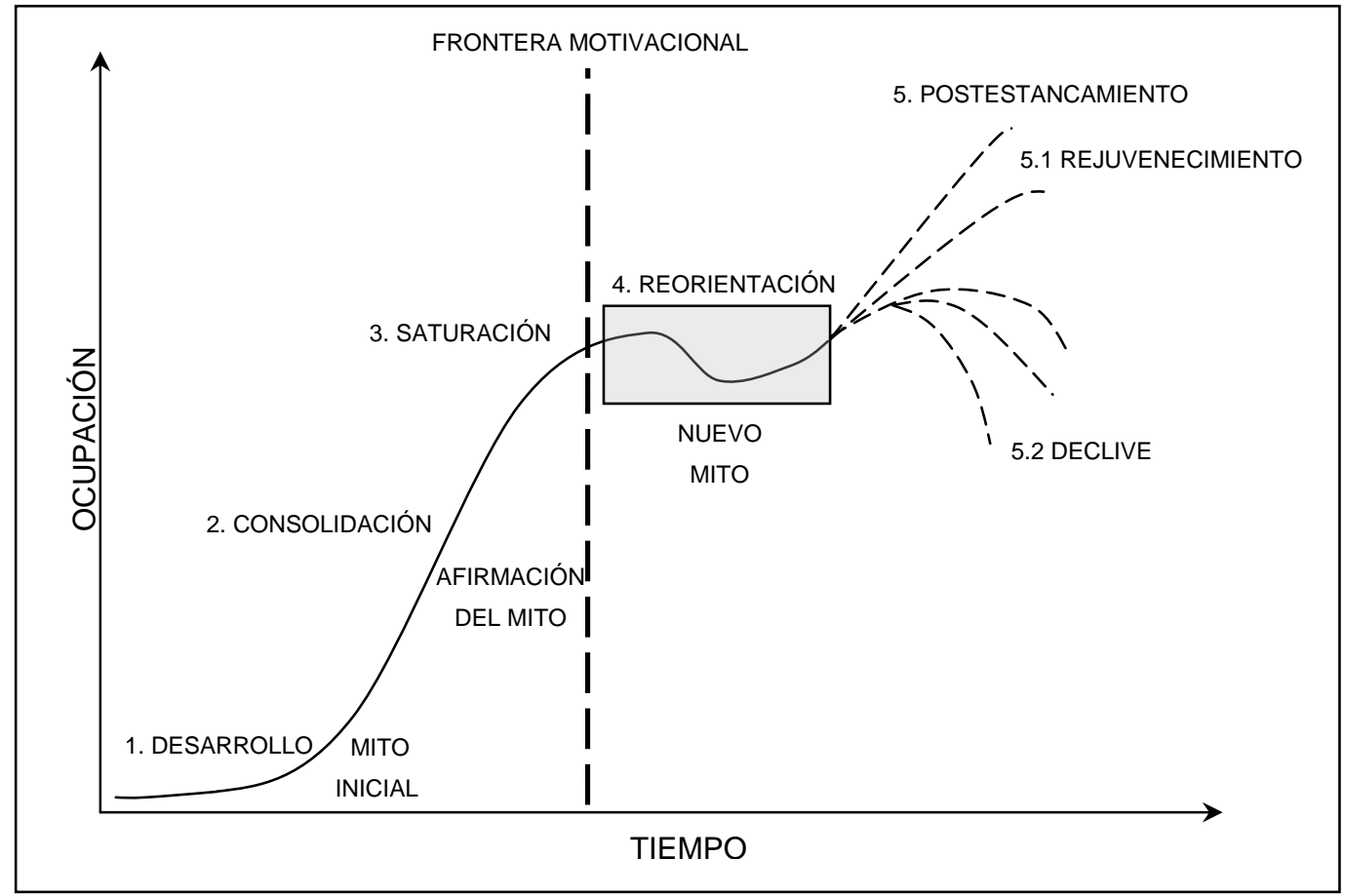

Fuente: Elaboración a partir de Agarwal $(2002,2006)$ y Chadefaud (1987). 


\subsection{La reorientación de los destinos}

Debido a que uno de los objetivos particulares de esta investigación hace referencia a la identificación de actuaciones estratégicas para mejorar la competitividad del destino, en el modelo se incorporó la idea de Agarwal (2002) relativa a la etapa de reorientación en los destinos.

La reorientación se instrumenta a través de estrategias y actuaciones asociadas a la competitividad y a la sostenibilidad. En el cuadro 1, se indica un conjunto de descriptores que permitieron determinar si Bahías de Huatulco experimenta una fase de reorientación.

Cuadro 1. Variables asociadas a la reorientación de destinos turísticos

\begin{tabular}{|c|c|}
\hline \multicolumn{2}{|r|}{ Competitividad } \\
\hline Estrategia & Actuación \\
\hline $\begin{array}{l}\text { Diversificación de la oferta a través de } \\
\text { recursos singulares. }\end{array}$ & $\begin{array}{l}\text { Puesta en valor de recursos naturales y culturales en el } \\
\text { destino o los alrededores. }\end{array}$ \\
\hline $\begin{array}{l}\text { Incorporación de atracciones } \\
\text { artificiales. }\end{array}$ & $\begin{array}{l}\text { Casinos, marinas deportivas, espacios hiperreales, parques } \\
\text { temáticos, etc. }\end{array}$ \\
\hline Mejora en la calidad del servicio. & Sistemas de calidad, entrenamiento y flexibilidad laboral. \\
\hline Accesibilidad. & $\begin{array}{l}\text { Enlace con los mercados emisores y conectividad a escala } \\
\text { local. }\end{array}$ \\
\hline Especialización. & $\begin{array}{l}\text { Aprovisionamiento de instalaciones y servicios especiales } \\
\text { para ciertos segmentos. }\end{array}$ \\
\hline Reposicionamiento. & Renovación del alojamiento y de la imagen. \\
\hline Adaptación. & Estudios para pronosticar tendencias futuras. \\
\hline Colaboración. & $\begin{array}{l}\text { Iniciativas entre los sectores público y privado para impulsar } \\
\text { el turismo. }\end{array}$ \\
\hline \multicolumn{2}{|r|}{ Sostenibilidad } \\
\hline Estrategia & Actuación \\
\hline $\begin{array}{l}\text { Recuperación de la calidad ambiental y } \\
\text { revitalización de áreas en declive. }\end{array}$ & $\begin{array}{l}\text { Medidas medioambientales, Agendas 21, ecoetiquetas, } \\
\text { capacidad de carga, entre otras. }\end{array}$ \\
\hline $\begin{array}{l}\text { Nuevas pautas a la creación de oferta, } \\
\text { límites al crecimiento y } \\
\text { reordenamiento de áreas saturadas. }\end{array}$ & $\begin{array}{l}\text { Planeamiento del uso de suelo, recalificación de suelos, } \\
\text { esponjamiento. }\end{array}$ \\
\hline
\end{tabular}

\section{RESULTADOS}

\subsection{El origen del destino}

Bahías de Huatulco se localiza a lo largo de la franja litoral del municipio de Santa María Huatulco, Oaxaca (figura 2). El sitio donde actualmente se ubica fue descubierto en 1969 por una delegación de funcionarios del Banco de México que en aquel entonces exploraban las costas del país con el objetivo de detectar sitios con potencial turístico. Un año después un grupo de técnicos gubernamentales fue destacado en la zona para realizar un reconocimiento general después del cual, durante los siguientes siete años, se llevaron a cabo estudios diversos para planificar el desarrollo del centro vacacional (Fernández, 1999:35-36). 
Figura 2. Ubicación del Centro Integralmente Planeado (CIP) Bahías de Huatulco.

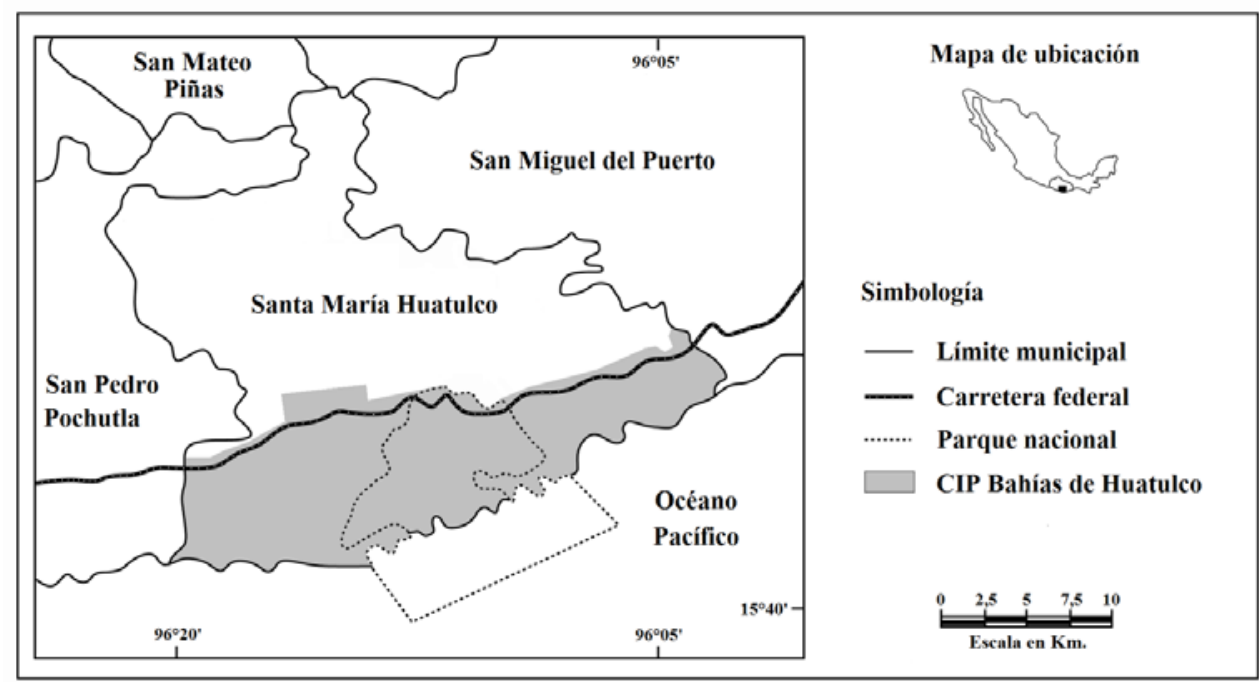

Fuente: Elaboración propia.

El 28 de mayo de 1984 el gobierno federal, a través de la hoy extinta Secretaría de Desarrollo Urbano y Ecología expropió a favor del Fondo Nacional de Fomento al Turismo (FONATUR), por causa de utilidad pública, un predio de 20.975 hectáreas al municipio de Santa María Huatulco, con la finalidad de disponer del suelo necesario para desarrollar un centro turístico (Diario Oficial de la Nación, 1984). De forma similar al caso de Cancún, el nuevo destino sería orientado al mercado internacional con la intensión de fomentar cuatro objetivos estratégicos: la captación de divisas, la inversión extranjera en el destino, el fomento al empleo y el impulso al desarrollo regional.

De acuerdo con el Plan Maestro de Bahías de Huatulco, el destino lograría afianzar su función turística a lo largo de tres etapas: desarrollo (1984-1988), consolidación (1988-2000) y saturación (2000-2018). Cada fase estaría asociada a metas estratégicas concernientes con el número de cuartos, el aforo turístico, el incremento de la población local y la ocupación de los diferentes usos de suelo (FONATUR, 1997).

\subsection{El ciclo de vida de Bahías de Huatulco}

Entre 1984 y 1988 se presentaron en Bahías de Huatulco la mayoría de los indicadores que, según Butler, evidencian una fase de desarrollo. Durante esa etapa el destino experimentó un intenso crecimiento soportado por la construcción de infraestructuras de cabeza y de servicios turísticos.

Durante 1988 y 2000 se manifestaron todos los rasgos que Butler asocia a la fase de consolidación, sin embargo las constantes fluctuaciones en el aforo turístico, así como el incumplimiento de las metas relacionadas con el número de cuartos construidos y con la estructura de los flujos turísticos (predominio de turistas nacionales), ponen al descubierto que en ese periodo hubo un crecimiento ralentizado que no permitió consumar la consolidación. 
Durante esta etapa el FONATUR encargó una encuesta para valorar la experiencia de los turistas en el destino. Los resultados obtenidos indicaron que Bahías de Huatulco no cubría las expectativas del 30\% de los visitantes (FONATUR, 1997:195) razón por la cual se asume que, hacia finales del siglo XX, el mito alrededor de los espacios litorales comenzaba a cambiar y en consecuencia la fórmula estandarizada de "sol, arena y mar" perdía fuerza de atracción.

Pese al crecimiento en la recepción turística, la ocupación promedio durante la segunda etapa se mantuvo por debajo del $60 \%$ y el número de turistas internacionales representaba apenas el $25 \%$ del volumen total captado por el destino.

En 1997 se llevó a cabo una revisión de la planificación del destino que condujo a la definición de un programa de reposicionamiento en el cual se marcaron varias metas para hacer coincidir la oferta del destino con las motivaciones vigentes (el mito, según Chadefaud) en el turismo litoral (FONATUR, 1997:193-211). Entre las metas señaladas destacan las siguientes:

- Imagen vinculada a la calidad ambiental, la sostenibilidad y a productos de playa, pesca, golf, cruceros, náutica, ecoturismo, convenciones y cultura.

- Promoción del alojamiento en modalidad de tiempo compartido para incentivar el aforo de viajeros a lo largo del año y con ello promover la venta de asientos de avión para asegurar la conexión aérea al destino.

- Incremento del número de cuartos hasta alcanzar los 4.000 en 2010.

- Incremento del transporte de pasajeros (aéreo, terrestre y marítimo).

- Mayor promoción y venta en el extranjero.

- Diseño de producto y comercialización especialmente a través de Internet.

Durante la tercera etapa (2000-2018) solamente se ha registrado un rasgo asociado, según el modelo de Butler, a la fase de estancamiento (la construcción de atracciones artificiales, a saber, una marina deportiva). Por ello se puede suponer que la evolución del destino no se aproxima a la fase de madurez. Esto se evidencia, además, al considerar que la ocupación promedio anual no ha logrado alcanzar-desde hace 22 años- niveles superiores al $57 \%$.

A inicio de esta etapa el Comité de Iniciativa Ciudadana, una agrupación local compuesta por inversionistas, empresarios y gestores públicos formuló el Plan de Gran Visión de Desarrollo Sustentable 2025, en el cual se establecieron pautas para potenciar la sostenibilidad, la inversión, la diversificación de oferta, así como la promoción y la comercialización del destino en nichos clave del mercado. Más tarde, ese mismo documento se constituiría en la base del Renacimiento de Huatulco, una estrategia de reorientación lanzada en 2008 desde la Presidencia de la República.

Las acciones emprendidas, primero por el FONATUR y después en el marco del Renacimiento Huatulco sugieren que desde hace aproximadamente 10 años el destino se encuentra en una fase de reorientación que busca superar el rezago en materia de oferta hotelera, inmobiliaria y recreativa, así como en la operación de rutas aéreas. Lo anterior 
permite corroborar el planteamiento de Agarwal (2002) relativo a la posible manifestación de fases de reorientación aún cuando los destinos no hayan experimentado el estancamiento o la saturación.

Al representar gráficamente el ciclo de vida del destino a través del modelo propuesto en este trabajo (figura 3) se observa que durante la segunda etapa de desarrollo (correspondiente con la fase de consolidación), ya se manifestaba un escenario que podría clasificarse de estancamiento debido al comportamiento de los niveles de ocupación cuyo valor fluctuaría entre el $43 \%$ y el $56 \%$.

Figura 3. El CVAT de Bahías de Huatulco según el modelo propuesto.

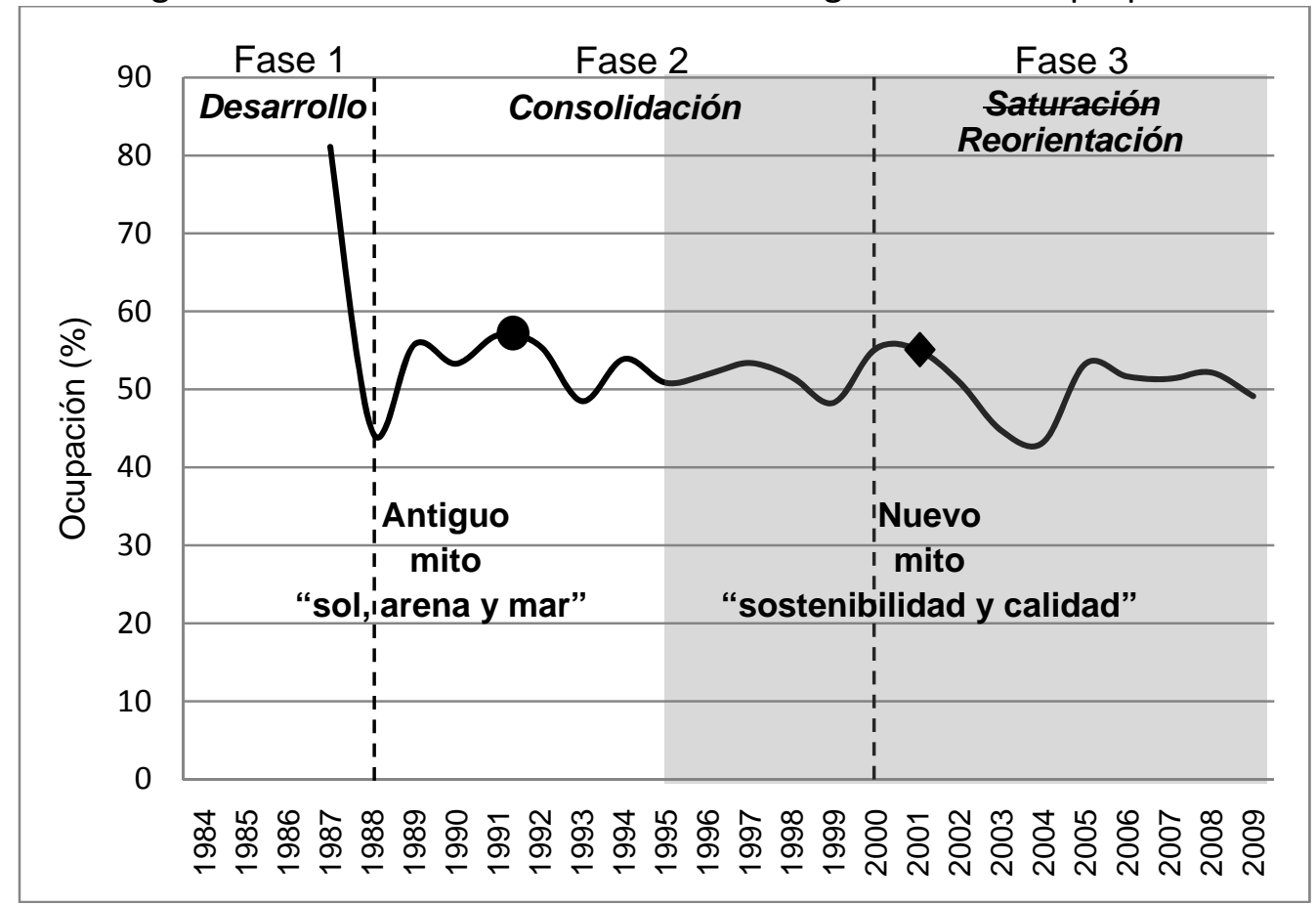

Fuente: Elaboración con base en FONATUR (2009) y Secretaría de Turismo de Oaxaca (2010).

El escenario detectado a través del modelo anterior es, igualmente, advertido mediante la representación del CVAT con el modelo de Butler (figura 4) en el cual se observa que a inicios de los años noventa se interrumpe la tendencia de crecimiento seguida por el destino.

Si ambos modelos (figuras 3 y 4) son capaces de señalar momentos críticos en la evolución de los destinos, ¿cuál de los dos es más adecuado? 
Figura 4. El CVAT de Bahías de Huatulco según el modelo Butler.

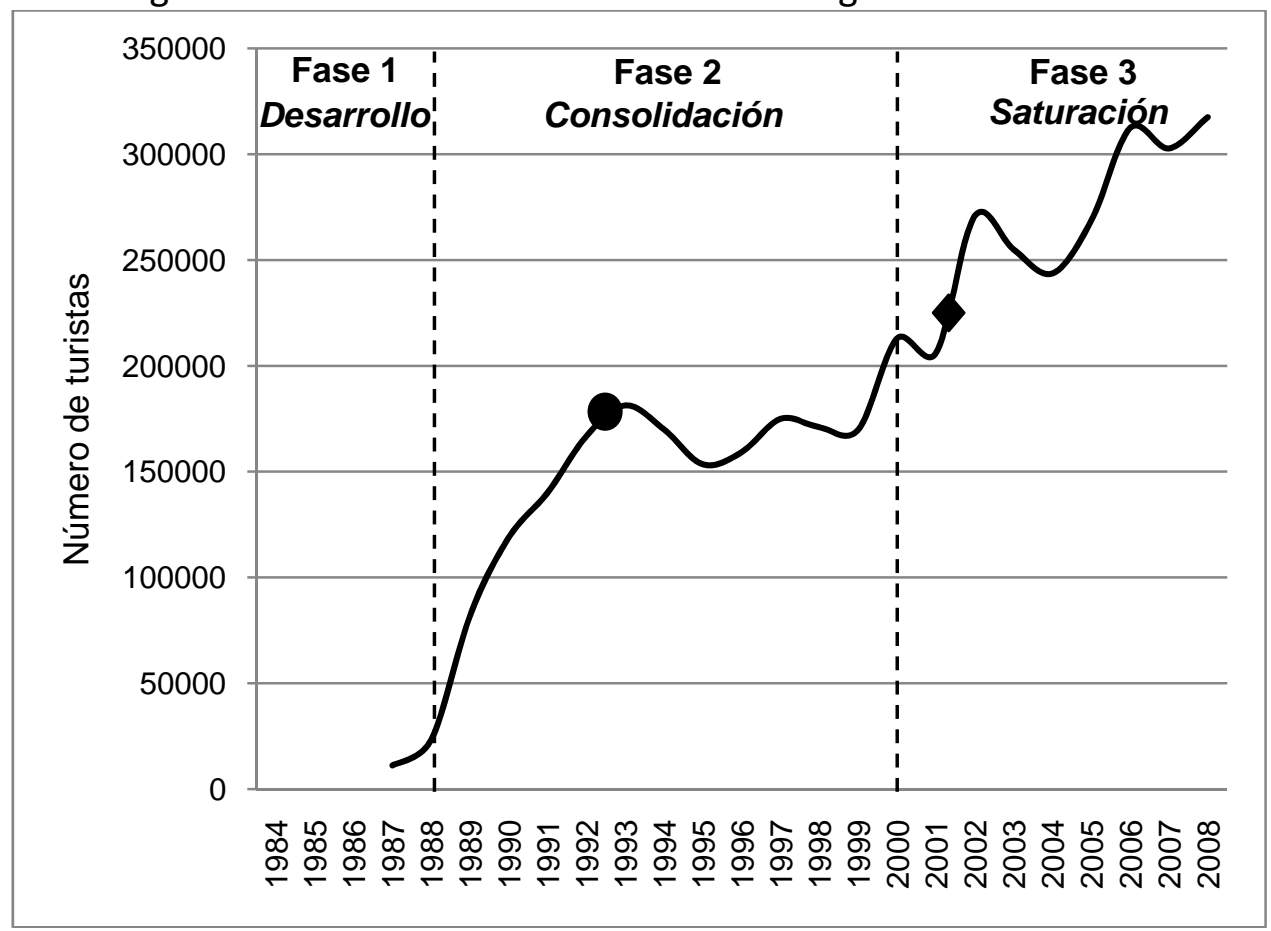

Fuente: Elaboración con base en FONATUR (2009).

Una primera aproximación a la cuestión revelaría que ambas propuestas tienen el mismo potencial para reflejar la situación del destino. Para demostrar lo anterior basta observar el círculo negro que aparece en las dos figuras y que señala, entre 1991 y 1992, que la situación competitiva de Bahías de Huatulco mostraba una tendencia positiva visible tanto en el orden de la ocupación como en el número de turistas. Parecería, entonces, que medir el ciclo de vida con una u otra variable implica los mismos alcances analíticos. Esta idea, sin embargo, cambia al centrar la atención en la tercera fase de desarrollo en la cual se percibe, hacia el año 2002, un comportamiento discordante entre las variables (ver la posición de los rombos color negro). Mientras que la magnitud alcanzada por el número de turistas refleja una tendencia de crecimiento que sugiere una situación positiva, el comportamiento seguido por la ocupación da cuenta de una caída que se acentúa en los años siguientes. De cara a esta situación, surge la preguntar acerca de cuál de los dos modelos resultaría más confiable para formular un diagnóstico y guiar la gestión.

Aún cuando ambos modelos trabajan con una visión reduccionista de la realidad, se asume que el ciclo de vida basado en la ocupación hotelera es más conveniente por dos razones: En primer lugar la ocupación refleja el desempeño promedio del destino a lo largo del año. Una ocupación elevada señalaría un consumo constante del alojamiento turístico (en temporada alta y baja) y por consecuencia un posicionamiento efectivo en el mercado. El manejo del número de turistas como variable de referencia, por su parte, no permite tal interpretación debido a que un incremento en el volumen alcanzado podría ser el resultado de una alta concentración de visitantes sólo en temporadas vacacionales. 
En segundo lugar el modelo propuesto en este trabajo permite al gestor turístico identificar escenarios y definir actuaciones oportunas que no dependen únicamente de una variable como ocurre en el modelo de Butler. En el siguiente cuadro se ejemplifican seis posibles escenarios definidos a través de dos indicadores (ocupación y número de cuartos) asociados al modelo alternativo.

Cuadro 3. Diagnóstico y gestión según el modelo de CVAT propuesto.

\begin{tabular}{|c|l|l|l|l|}
\hline Escenario & Ocupación & $\begin{array}{c}\text { Cambio en el } \\
\text { núm. de cuartos }\end{array}$ & \multicolumn{1}{|c|}{ Diagnóstico } & \multicolumn{1}{|c|}{ Medidas de gestión } \\
\hline 1 & Sin cambio & Mayor & $\begin{array}{l}\text { El destino, de hecho, ha } \\
\text { incrementado su } \\
\text { competitividad. }\end{array}$ & $\begin{array}{l}\text { Fortalecer estrategias } \\
\text { vigentes. }\end{array}$ \\
\hline 2 & Sin cambio & Menor & $\begin{array}{l}\text { Pérdida de } \\
\text { competitividad. Los } \\
\text { hoteles que se retiran del } \\
\text { destino se llevan una } \\
\text { parte del mercado. }\end{array}$ & $\begin{array}{l}\text { Necesidad de fortalecer la } \\
\text { imagen del destino. Asociar } \\
\text { la imagen del centro } \\
\text { turístico al mito vigente. }\end{array}$ \\
\hline 3 & Menor & Mayor & $\begin{array}{l}\text { Efecto normal derivado } \\
\text { del incremento de la } \\
\text { oferta de alojamiento. }\end{array}$ & $\begin{array}{l}\text { Marketing y reforzamiento } \\
\text { de los canales de } \\
\text { distribución. }\end{array}$ \\
\hline 4 & Menor & Igual/menor & $\begin{array}{l}\text { Efecto derivado de la } \\
\text { presencia de destinos } \\
\text { sustitutivos. }\end{array}$ & $\begin{array}{l}\text { Iniciar fase de reorientación } \\
\text { y aplicar estrategias de } \\
\text { reestructuración ligadas al } \\
\text { mito vigente. }\end{array}$ \\
\hline 5 & Mayor & Igual/mayor & $\begin{array}{l}\text { El destino ha fortalecido } \\
\text { su competitividad. }\end{array}$ & $\begin{array}{l}\text { Seguimiento a las acciones } \\
\text { que promovieron llegar al } \\
\text { nivel de ocupación } \\
\text { reportado. }\end{array}$ \\
\hline 6 & Mayor & Menor & $\begin{array}{l}\text { Consecuencia esperada de } \\
\text { la redistribución de la } \\
\text { demanda entre los cuartos } \\
\text { que aún operan. }\end{array}$ & $\begin{array}{l}\text { Vigilar que el número de } \\
\text { turistas no disminuya. }\end{array}$ \\
\hline
\end{tabular}

De acuerdo la figura 3 y cuadro 3, desde mediados de los años noventa la situación de Bahías de Huatulco ha transitado por los escenarios 1, 3 y 5 . Ello explica que a inicios del presente siglo se constituyera el Consejo de Iniciativa Ciudadana de cuyo trabajo derivaría el Plan de Gran Visión de Desarrollo Sustentable Huatulco 2025, a partir del cual iniciarían las acciones relacionadas con la fase de reorientación y de las cuales se aporta un resumen en la siguiente sección.

\subsection{La reorientación de Bahías de Huatulco}

Las estrategias de reestructuración comenzaron a perfilarse a comienzo de la pasada década. De acuerdo con la información colectada a través de entrevistas a representantes de entidades de gobierno, empresariales y civiles locales; y mediante la revisión de fuentes secundarias (Fernández, 1999; Brenner, 2005; Vázquez, 2005 y prensa local), se identificaron 91 actuaciones de reestructuración implementadas en el destino entre enero de 2003 y julio de 2010; de las cuales 72 mostraron vinculación con el ámbito de la competitividad y 19 con 
la sostenibilidad. Una descripción detallada de las mismas es aportada por López (2010:7789).

Entre las acciones encausadas para mejorar la competitividad del destino se destacan las siguientes: iniciativas para el uso público recreativo del Parque Nacional Huatulco, apertura de la zona eco-arqueológica de Copalita, construcción de una marina deportiva y un puerto de cruceros, organización de eventos deportivos internacionales, certificación de la calidad hotelera, certificación de prestadores de servicios, creación de fondos de garantía, riesgo compartido y promoción conjunta entre aerolíneas y el destino para promover el tránsito aéreo desde ciudades de Norteamérica y Reino Unido; expansión de la pista del aeropuerto, promoción a la inversión hotelera e inmobiliaria, renovación de instalaciones hoteleras para incrementar categoría y creación de un equipo de trabajo para la fundación de la Oficina de Convenciones y Visitante.

En cuanto a la sostenibilidad destacan la instauración de los comités Agenda 21 Local y playas limpias, la participación a nivel municipal en el Comité de Cuenca de la Costa de Oaxaca, así como la creación de una agrupación (Equipo Verde, Asociación Civil.) cuyo trabajo ha permitido certificar al destino como comunidad sostenible mediante los esquemas de Green Globe 21 y Earth Check.

Al comparar las actuaciones antes señaladas con la información mostrada en el cuadro 1, queda claro que las entidades de gobierno y la sociedad civil de Bahías de Huatulco han ejecutado acciones asociadas a cada una de las estrategias de reestructuración. No obstante, mediante el trabajo de campo y la consideración de elementos del macroambiente, se han identificado algunas áreas de oportunidad vinculadas a las estrategias de especialización y adaptación que al ser atendidas podrían coadyuvar en la reorientación del destino.

De acuerdo con Yeoman (2008), se recomienda el diseño de productos asociados a perfiles sociodemográficos emergentes, específicamente dirigidos al segmento senior y a las familias monoparentales, debido a la evolución poblacional y al incremento de separaciones en países de Norteamérica (http://cuentame.inegi.gob.mx/poblacion/myd,aspx?tema=P; National Center for Health Statistics, 2010).

Mediante una investigación exploratoria se corroboró que la mayoría de los hoteles del destino no aprovechan las funciones relacionales, de marketing y gestión de la reputación que ofrece la Web 2.0. Según Jiménez (2009) Internet es el medio donde el $84 \%$ de las personas buscan información sobre viajes, por esta razón se asume necesario dinamizar las funciones antes señaladas en las páginas web de los hoteles así como en las redes sociales en donde los establecimientos tienen presencia.

Se recomienda, además, considerar la puesta en marcha de un observatorio turístico encargado de coordinar la definición y monitoreo de variables clave para el desarrollo de la actividad y de valorar los efectos de las medidas emprendidas. Finalmente cabe hacer mención que los esfuerzos por avanzar hacia la sostenibilidad en el destino se han 
concentrado en las dimensiones ambiental y económico-empresarial sin prestar la misma atención al ámbito social. Sobre este tema deben fomentarse futuras actuaciones.

\section{CONCLUSIONES}

Ante la necesidad, por un lado, de dar seguimiento al ciclo de vida de los destinos turístico $y$, por otra parte, de perfeccionar los modelos para conducir el análisis, en este trabajo se propuso una versión modificada del CVAT para examinar la situación de los destinos ex novo.

El modelo propuesto y aplicado en Bahías de Huatulco permitió el reconocimiento de una fase de reorientación en el destino misma que se corroboró en la investigación de campo al identificar la presencia de una amplia gama de actuaciones de reestructuración. Los resultados arrojados por la investigación permiten suponer que el modelo aplicado muestra ventajas respecto al CVAT propuesto originalmente por Butler, sin embargo, es necesario hacer notar que ambos modelos manejan una visión reduccionista del desarrollo por el hecho de basar su análisis en el examen de una sola variable (ocupación y número de turistas respectivamente).

Debido a lo anterior es necesario reorientar la concepción sobre el ciclo de vida y sus modelos de medición. En relación con ello se destaca la pertinencia de retomar y ensayar algunas propuestas que intentan aproximar las ideas del ciclo de vida y la sostenibilidad, entre las cuales se pueden señalar a Priestley y Mundet (1998:106-107) y más recientemente a López (2010:94-97) cuyo modelo propone la integración de la metodología de la Agenda 21 Local a la gestión y monitoreo del ciclo del área turística.

\section{REFERENCIAS BIBLIOGRÁFICAS}

AGARWAL, S. (1997). "The Resort Cycle and Seaside Tourism: An Assessment of its Applicability and Validity". Tourism Management, 18 (2), 65-73.

AGARWAL, S. (2002). "Restructuring Seaside Tourism. The Resort Lifecycle". Annals of Tourism Research, 29 (1), 25-55.

AGARWAL, S. (2006). "Coastal Resort Restructuring and the TALC". In BUTLER, R. (ed.). The tourism Life Cycle. Vol. 2. Conceptual and Theoretical Issues. Clevendon: Channel View.

ANTÓN, S. (2004). “De los procesos de diversificación y cualificación a los productos turísticos emergentes. Cambios y oportunidades en la dinámica reciente del turismo litoral". Papeles de Economía Española, 12, 316-333.

ARTIGUES, A. (2001). "Turismo de espacios litorales e insulares", En BARRADO, D. y CALABUIG, J. (ed.). Geografía mundial del turismo. Madrid: Síntesis.

BARBOSA, A. (2007). "Estudio comparativo de playas: Mazatlán, Acapulco, Cancún y Los Cabos (1970-2005)". II Congreso Internacional de Investigación y Docencia. Universidad Autónoma de Durango. 
BRENNER, L. (2005). "State-Planned Tourism Destinations: The Case of Huatulco, Mexico" Tourism Geographies, 7 (2), 138-164.

BUTLER, R. (1980). "The Concept of Tourism Area Cycle of Evolution: Implications for Management of Resources". In BUTLER, R. (ed.) (2006). The Tourism Life Cycle. Vol. 1. Applications and Modifications. Clevendon: Channel View.

CALLIZO, J. (1989). "El espacio turístico de Chadefaud, un entreverso teórico: del historicismo al materialismo dialéctico y el sistemismo behaviourista". Geographicalia, 26:37-44.

CAMISÓN, C. y MONFORT, V. (1998). "Estrategias de reposicionamiento para destinos turísticos maduros: el caso de la Costa Blanca". Estudios Turísticos, 135, 5-28.

CLEGG, A. and ESSEX, S. (2000). "Restructuring in Tourism: The Accommodation Sector in a Major British Coastal Resort". International Journal of Tourism Research, 2 (2), 77-95.

COHEN-HATTAB, K. and SHOVAL, N. (2004). "The Decline of Israel's Mediterranean Resorts: Life Cycle Change versus National Tourism Master Planning". Tourism Geographies, 6 (1), 59-79.

COOPER, C. (1994). "The Destination Life Cycle: an update". In SEATON, A. (ed.). Tourism. The State of the Art. Chichester: Wiley.

DIARIO OFICIAL DE LA NACIÓN (1984). "Decreto Expropiatorio de la comunidad denominada Santa María Huatulco, municipio de Santa María Huatulco, Estado de Oaxaca". 29 de mayo.

DIEDRICH, A. and GARCIA-BUADES, E. (2009). "Local Perceptions of Tourism as Indicators of Destination Decline". Tourism Management, 30 (5), 512-521.

DONAIRE, J. y MUNDET, LI. (2001). "Estrategias de reconversión turística de los municipios litorales catalanes". Papers de Turisme, 29, 51-65.

FERNÁNDEZ, E. (1999). Bahías de Huatulco. Sol y sombras. Tesis de licenciatura. Universidad Nacional Autónoma de México.

FONDO NACIONAL DE FOMENTO AL TURISMO (1997). Estrategias de reposicionamiento del Desarrollo Huatulco, Oaxaca. México: FONATUR.

FONDO NACIONAL DE FOMENTO AL TURISMO (2009). Datos básicos Huatulco. Gerencia de Diseño de Planes de Desarrollo. México: FONATUR.

GETZ, D. (1992). "Tourism Planning and Destination Life Cycle". Annals of Tourism Research, 19: 752-770.

JIMÉNEZ, R. (2009). “¿Cómo influyen las redes sociales en el turismo?”. En Encuentro café 2.0. Las redes sociales y el turismo. SEGITTUR. Disponible en: http://www.segittur.es/content/view/full/1152//\%28offset\%29/20

JOHNSTON, C. (2001). "Shoring the Foundations of the Destination Life Cycle Model, Part 1: Ontological and Epistemological Considerations". Tourism Geographies. 3 (1), 2-28.

JORDAN, P. (2000). "Restructuring Croatia's Coastal Resorts: Change, Sustainable Development and the Incorporation of Rural Hinterlands". Journal of Sustainable Tourism. 8 (6), 525-539.

LÓPEZ, V. M. (2010). La reorientación en los destinos litorales planificados. Caso de estudio: Bahías de Huatulco, Oaxaca (México). Proyecto fin de master. Universidad de Alicante. Disponible en: http://rua.ua.es/dspace/handle/10045/14977 
MANENTE, M. and PECHLANER, H. (2006). "How to Define, Identify and Monitor the Decline in Tourist Destinations: Towards an Early Warning System", In Butler, R. (ed.). The Tourism Life Cycle. Vol. 2. Conceptual and Theoretical Issues. Clevendon: Channel View.

MONCADA, P. (2008). "Renovarse o morir, el desarrollo histórico de Cancún revisado a través del modelo de ciclo de vida del área turística de Butler". Boletín Turístico de Cancún, 1 (1), 3-7.

NATIONAL CENTER FOR HEALTH STATISTICS (2010). National Vital Statistics Report. United States of America, Hyattsville: National Vital Statistics System, 58, 25.

PRIESTLEY, G. and MUNDET, LI. (1998). "The Post-Stagnation Phase of the Resort Cycle". Annals of Tourism Research, 25 (1):85-111.

SANTAMARÍA, A. y BARBOSA, A. (2008). “Ciclo turístico en Mazatlán. Fase de exploracióninvolucramiento-desarrollo (1970). Estancamiento o crisis sistémica (19922004)" Topofilia, 1 (1). Disponible en http://topofilia.net/santamaria.html

SECRETARÍA DE TURISMO DE OAXACA (2010). Indicadores básicos de la actividad turística en el estado de Oaxaca, 2009. Oaxaca: SECTUR.

VÁZQUEZ, V. (2005). Las dependencias regionales y globales de la economía turística de Bahías de Huatulco. Tesis de doctorado. Universidad Nacional Autónoma de México.

VERA, F. (2004). "Desarrollo turístico y control del crecimiento: utilidades de la capacidad de carga". En Pardellas, X. (dir.): Potencial turístico de territorios periféricos. Universidad de Vigo.

VERA, F.; LÓPEZ, F.; MARCHENA, M. y ANTÓN, S. (1997). Análisis territorial del turismo. Una nueva geografía del turismo. Madrid: Ariel.

VERA, F. and RODRÍGUEZ, I. (2010). "Tourism Strategies for the Renovation of Mature Coastal Destinations in Spain". In IV International Conference on Sustainable Tourism. New Forest, United Kingdom.

VIRGEN, C. (2009). "El ciclo de vida de un destinos turístico: Puerto Vallarta, Jalisco, México". Cultur, 3 (1), 1-25.

YEOMAN, I. (2008). Tomorrow's Tourist. Scenarios \& Trends. Amsterdam: Elsevier.

\section{AGRADECIMIENTO}

El autor agradece el apoyo concedido por la Fundación Carolina a través de una beca de postgrado

\section{Nota:}

El presente documento forma parte de los resultados parciales asociados al proyecto CSO-00613 MICINN sobre Renovación de Destinos Turísticos Consolidados de Litoral (RENOVESTUR), ejecutado por el Instituto Universitario de Investigaciones Turísticas de la Universidad de Alicante, España. ( http://blogs.ua.es/renovestur/) ). 